\title{
Strong Electromagnetic Pulse Comprehensive Protection Methods Research for UAV
}

\author{
Baozhou Du, Yazhou Chen, Erwei Cheng \\ Institute of Electrostatic \& Electromagnetic Protection, Ordnance Engineering College, \\ Shijiazhuang 050003, China
}

\author{
Keywords: HEMP; UWB Electromagnetic Pulse; Electromagnetic Protection Methods; Sensitive \\ devices.
}

\begin{abstract}
Data link system, as important guarantee for unmanned aerial vehicle equipment to complete its battlefield function, its anti-electromagnetic pulse capacity interference directly affect the safety of airborne communications. Strong electromagnetic pulse has become an important source of electromagnetic interference with its high power, wide frequency band and other characteristics, which brings huge threat to the internal electronic equipment and devices of UAV. Therefore, a new method of electromagnetic pulse field protection for space electromagnetic pulse field protection and sensitive devices in circuit is presented, and the method of electromagnetic pulse field-path integrated protection for sensitive devices is discussed. The use of electromagnetic protection method, protect the internal sensitive modules from the electromagnetic pulse voltage or current which Coupling to the circuit. And the insertion loss of the protection module is small, does not affect the normal operation of the circuit.
\end{abstract}

\section{Introduction}

With the rapid development of modern science and technology, it makes the development trend of the future war gradually evolved into high-tech local war under the condition of information technology [1]. The success application of new equipment, new concept weapon in battlefield, profoundly changed the meaning of the war as was known in the past, it makes the electromagnetic environment in the battlefield become more complicated. At the same time, weapons and equipments are facing acid tests and challenges.

During several worldwide high-tech local war fares in the recent years, information weapon equipments gradually replace traditional weapons and equipments, which become a weapon for winning the war. Unmanned aerial vehicle (UAV) got the favor of the military, with its high mobility, zero casualties and high cost effectiveness, ways of landing agile diversity, unique advantages, beyond that, it has broad application prospects [2]. Because of the limiting factors for design needs, such as the distribution inside the fuselage with a large number of electronic equipment in narrow space of UAV, the distribution of internal electromagnetic sensitive components is concentrated, the strong electromagnetic environment has great threat on the highly integrated drone as a result.

According to the regulations of the international electro technical commission's standard, high power electromagnetic environment refers to the electromagnetic field intensity is higher than 100 $\mathrm{V} / \mathrm{m}$ environments [3]. Strong electromagnetic pulse, for example, ultra-wideband electromagnetic pulse, since it has a steep rise time and pulse width (all in a couple of nanoseconds or even picoseconds order of magnitude and release high intensity pulse power (tens of GWs or even hundreds of GWs) instantaneously, it can break through many protective circuit, interfere the normal communication and even damage electronic devices. It has become an urgent need to carry out the strong electromagnetic pulse protection for UAV as magnetic sensitive equipment. Some domestic related units research electromagnetic pulse interference problems for UAV in recent years. Chinese ships reach center, Zheng ShengQuan, put forward several kinds of protection for antenna coupling space electromagnetic pulse field and the sensitive device in the circuit, a new method of electromagnetic pulse road protection, discusses the electromagnetic pulse field to road of sensitive system design of comprehensive protection [4-5]. Ordnance Engineering College, Chen YaZhou 
group proceed the study of the continuous wave irradiation effect of UAV, obtain the frequency which make the UAV data link communication failure and sensitivity threshold was founded, using electromagnetic simulation and injection test analyzes the failure mechanism of the UAV data link [6]. This paper will focus on the strong electromagnetic pulse protection method for UAV. Due to the limited impulse power affordability of the existing limiter devices, it is easy to be damaged by the strong electromagnetic pulse signal coupled by the antenna. On the other hand, a single protection method is not sufficient to provide adequate protection for the sensitive modules of the device. Therefore, this paper proposes the need for field-to-road integrated protection design for the electromagnetic pulse field and the conducting electromagnetic pulse in the circuit for UAV.

\section{Major strong electromagnetic pulse classification}

\subsection{HEMP}

Nuclear electromagnetic pulse generally refers to the high-altitude nuclear explosion electromagnetic pulse, which is generally as a plane wave processing near the ground, in a certain region of its pulse waveform can be approximated as a double exponential waveform. IEC latest promulgated HEMP double index waveform parameter standard provides the pulse front rise time is $1.8-2.8 \mathrm{~ns}$, half wave width is $18-28 \mathrm{~ns}$, its standard parameters is higher than other standards in the past requirements The waveform shown in Figure 1 .

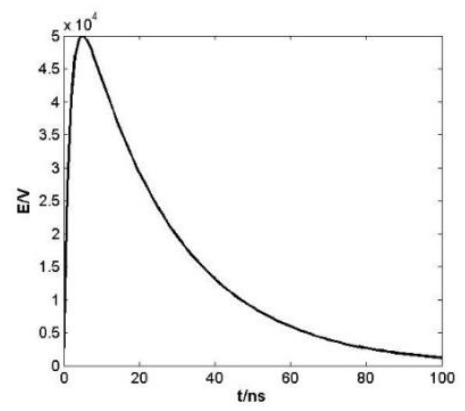

Fig. 1 Double exponential waveform according to IEC

The field strength for the time domain analysis formula is:

$$
E(\mathrm{t})=\mathrm{E}_{0} k\left(\mathrm{e}^{-\alpha t}-\mathrm{e}^{-\beta t}\right)
$$

$E_{0}$ is the peak field strength, $k$ is the correction coefficient, $\alpha, \beta$ respectively determine the falling edge and the rising edge time of the double exponential waveform.

The electromagnetic field of the HEMP has the characteristics of steep front, high peak, and wide frequency coverage. The radiation field can easily threaten the UAV system, especially the electromagnetic weak device; leading to the degradation of UAV performance and can not finish its combat task well.

\subsection{UWB electromagnetic pulse}

Ultra-wideband (UWB) electromagnetic pulse refers to the peak power greater than $100 \mathrm{MW}$, rising front for the sub-nanosecond or picoseconds order, the relative bandwidth of more than $25 \%$ of the electromagnetic pulse. Its spectrum can be from several tens to several $\mathrm{GHz}$ or several tens $\mathrm{GHz}$ UWB electromagnetic pulse frequency content in the microwave frequency band, so narrowband high-power microwave (HPM) as pervasive. Because of its wide bandwidth range, not only the back door coupling is easy, front door coupling problem is more difficult than the narrow band HPM. If the ultra-wideband (UWB) electromagnetic pulse can radiate enough energy and power, it is more damaging than the high-altitude nuclear explosion electromagnetic pulse. The ultra-wideband electromagnetic pulse referred to in this paper is a differential Gaussian pulse approximation. The field strength for the time domain analysis formula is:

$$
E(\mathrm{t})=\frac{t-t_{0}}{\tau} \exp \left[-\frac{4 \pi\left(t-t_{0}\right)^{2}}{\tau^{2}}\right]
$$

$t_{0}$ for the time delay, $\tau$ is the time decay constant. 


\section{Strong Electromagnetic Pulse Protection Method for UAV}

\subsection{Energy Coupling Analysis of Strong Electromagnetic Pulse for UAV}

Strong electromagnetic pulse can enter the electronic equipment through a variety of coupling channels, causing harm to electronic equipment, such as antenna, cable, cabin and chassis holes. However, which can lead to damage to electronic equipment, mainly through the sending and receiving of electromagnetic signals antenna coupling. After electromagnetic pulse enter into the sensitive device, it can make the device inside the local temperature rises sharply, causing fusing and stress damage. Injection test shows that: dozens of volts of electromagnetic pulse can be sensitive components damage.

\subsection{Principle Analysis of Electromagnetic Protection}

Through the analysis of section 2.1, ultra-wideband electromagnetic pulse enter into RF front-end circuit of UAV is mainly through the UAV's antenna coupling. After electromagnetic pulse enter into the UAV internal electromagnetic sensitive components, because of its instantaneous power is far greater than the device can withstand the maximum input power, the device can make a sharp rise in internal temperature, causing fusing and stress damage, Which led to UAV's data link cannot work properly.

Therefore, in order to limit or reduce the coupling into the UAV internal electromagnetic weak links of ultra-wideband electromagnetic pulse energy, from the protection of the principle point of view, we can refer the two methods on RF front-end:(1) reduce the coupling of the electromagnetic pulse energy to the antenna; (2) reduce the amplitude of electromagnetic pulse energy spread to the UAV RF front-end. The above two methods can be achieved by installing various types of limiters and filters in the UAV's internal circuit, and taking into account the limited power consumption of the existing limiter devices, it is easy to be damaged when high power electromagnetic pulse enter into limiter. On the other hand, a single electromagnetic protection method is not enough to protect the weak parts of the unmanned aerial vehicle. Therefore, the need for comprehensive use of electromagnetic protection integrated methods design of unmanned aerial vehicles

\subsection{Electromagnetic protection integrated design}

For the UAV internal electromagnetic sensitive components of ultra-wideband electromagnetic pulse integrated protection design, in addition to the establishment of the necessary and complete experimental facilities, the establishment of the relevant standards. At the same time, also need from the following aspects of the system to carry out UAV ultra-wideband electromagnetic pulse protection work [7]:

1) Do a good job of ultra wide band electromagnetic pulse on the UAV sensitive system, the device hazardous characteristics of the forecast work. Combined with the model test, make use of relevant electromagnetic simulation software for the relevant theoretical calculations, Predict Amplitude, Spectrum, Waveform Characteristics of Ultra-Wideband Electromagnetic Pulse Coupled by Antenna Before RF Front End Sensors in UAV accurately And compared with the maximum input power or damage threshold of the electromagnetic sensitive device to evaluate the damage degree of the UWB electromagnetic pulse to the UAV.

2) The development of electromagnetic pulse comprehensive protection for UAV's sensitive device In addition to the usual "front door" (antenna) protection, "back door" (power cables, holes, etc.) protection, but also from the overall, system, sub-systems, modules, devices and other links to carry out the comprehensive protection of electromagnetic pulse. Need to be addressed key and difficult issues include the study of new electromagnetic protective materials, structures, etc., developed a fast response time, large flow capacity of the protection period and modules. At the same time, also need to pay attention to protective measures can not affect the normal combat performance of UAVs.

3) Protection Test and Verification Test of Electromagnetic Protection Measures for Unmanned Aerial Vehicle. The test and verification method of the protective effect of UAV protection device, protection module and protection method are studied. Research and evaluation methods and the establishment of the corresponding index evaluation standard system and UWB electromagnetic pulse protection of UAV indicators included in the equipment, system design and acceptance requirements. 


\section{Conclusion}

It is urgent to carry out research on the electromagnetic protection of UAV. As the strong electromagnetic pulse with a fast rise time, radiation power and other characteristics of a single electromagnetic pulse protection method is difficult to fully and effectively on the UAV sensitive modules, devices protection need to use a variety of electromagnetic protection means for unmanned aerial vehicle integrated electromagnetic protection design to improve the survivability of UAV equipment in the future battlefield under complex electromagnetic environment condition.

\section{Reference}

[1] LIU Shang He. The weaponry electromagnetic environment effects and its development trend[J]. Journal of the Academy of Equipment Command and Technology, 005, 16(1): 1-6.

[2] Zhang Dong xiao, Chen Ya zhou, Tian Qing min, et a1. Lightning pulse magnetic field effects on UAV system. High Power Laser and Particle Beams, 2015, 27: 103236(5).

[3] GIRI D V, TESCHE F M. Classification of intentional electromagnetic environments(IEME)[J]. IEEE Transactions on Electromagnetic Compatibility, 2004, 46(3): 322-328

[4] LIU Peiguo, LIU Chenxi, TAN Jianfeng, et al. Analysis of the research development on HPM/EMP protection[J].Chinese Journal of Ship Research, 2015, 10(2):2-6.

[5] ZHENG Sheng quan, DENG Feng, WANG Dongdong et al. Overview of the HPM field-circuit integrated protection methods for electronic equipment and system RF-channels[J]. Chinese Journal of Ship Research20, 15, 10(2): 7-14.

[6] Zhang Dongxiao, ChenYazhou, Tian Qingmin, et al. Mechanism of continuous wave electromagnetic radiation on UAV's vice telecontrol system. High Power Laser and Particle Beams, 2015, 27:103237

[7] ZHENG Shengquan, WU Xiaoguang, ZHU Yingfu. Electromagnetic pulse treats to ship-flat and requirements of protection [J]. Journal of Microwaves, 2010, 26(Supp 2): 101-104. 